\title{
DIFFERENCES IN SIMULATED CAR FOLLOWING BEHAVIOR OF YOUNGER AND OLDER DRIVERS
}

\author{
Elizabeth Dastrup ${ }^{1}$, Monica N. Lees ${ }^{2}$, Jeffrey D. Dawson ${ }^{1}$, \\ John D. Lee ${ }^{2,3}$, Matthew Rizzo $2,3,4$ \\ ${ }^{1}$ Department of Biostatistics, College of Public Health \\ ${ }^{2}$ Department of Mechanical and Industrial Engineering \\ ${ }^{3}$ Public Policy Center \\ ${ }^{4}$ Department of Neurology, Carver College of Medicine \\ University of Iowa \\ Iowa City, IA, USA \\ Email: matthew-rizzo@uiowa.edu
}

\begin{abstract}
Summary: Older drivers are at risk for vehicle crashes due to impairments of visual processing and attention, placing these drivers at greater risk in driving tasks that require continuous attention to neighboring traffic, especially lead vehicles (LVs). We investigated car following behavior in 42 younger drivers (ages 18 to 44 years) and 58 older drivers (ages 65 to 86 years) in a driving simulator. The drivers were instructed to maintain two car lengths from a virtual LV. The LV varied its velocity according to a sum of three sine waves, making the velocity changes unpredictable to the drivers. A Fourier analysis was performed using the vehicle trajectory data to derive measures of coherence, gain, and delay as indices of car following behavior. These measures as well as headway distance were compared between the two groups. Older drivers were less able to match changes in the LV velocity indicated by lower coherence $(0.76 \mathrm{v}$. $0.84, p=0.019)$ and larger gain $(2.24$ v. $1.74, p=0.031)$. However, these drivers followed further behind the LV than younger drivers, a potential compensatory strategy that may reduce collision risk for older drivers.
\end{abstract}

\section{OBJECTIVE}

Simulation can be used to assess performance profiles in drivers who are at risk for a crash due to age related cognitive impairments. The underlying assumption is that performance in the simulator, which reflects perceptual, cognitive and motor limits of what drivers can do under controlled conditions, is related to driver behavior - what drivers actually do on the road. A key issue in studies of older drivers concerns the criteria for development of scenarios for testing at risk drivers. Useful scenarios are capable of assessing specific mechanisms of impairment and correlate well with real world driving performance and crash data in the epidemiologic record. In this particular study of older driver safety we focused on car following as a situation with the potential for high crash risk, using a simulator scenario that allowed us to capture a number of continuous measures of performance in drivers who are at risk for crashes due to age-related differences in driver vision, vigilance, cognition, judgment, motor abilities, and acceptance of risk.

In 2005, rear-end collisions accounted for 29.6 percent of all police reported crashes (National Highway Traffic Safety Administration, 2007). These types of collisions have largely been 
attributed to drivers adopting unsafe following distances and drivers engaging in non-driving related tasks while driving (Knipling et al. 1993, Wang et al. 1996, Dingus et al. 1997, 1998). Reacting to changes in the velocity of a lead vehicle (LV) is a ubiquitous task on the roads. Following too closely or not reacting quickly enough to abrupt changes made by a LV may lead to a crash. This paper compares car following behavior in younger and older drivers using a driving simulator. We hypothesized that older and younger drivers exhibit different driving behaviors on the road. Older drivers have more driving experience and hence they may make different choices, have different levels of vigilance, or react to driving challenges in different ways. Our methods and analyses were informed by the work of Brookhuis et al., 1994 using an instrumented vehicle, and Andersen and Ni, 2005 using a simulator (cf, Dastrup et al., in press; Brackstone et al., 1999).

\section{METHODS}

\section{Participants}

Driving performance during a car following scenario was evaluated for a group of younger drivers and a group of older drivers. Subjects were recruited from the community with an advertisement that was approved by the University of Iowa Institutional Review Board. We studied 19 female and 23 male younger drivers aged $18-44$ with an average age of 28.6 years $(\mathrm{SD}=7.68)$ and 22 female and 36 male elderly drivers aged $66-88$ with an average age of 74.8 $(\mathrm{SD}=4.97)$.

\section{Simulated Driving Performance}

All participants completed a car following scenario in SIREN (Simulator for Research in Ergonomics and Neuroscience), an interactive driving simulator that creates an immersive, virtual environment for assessing at-risk drivers in a health care setting (Rizzo et al., 2005). SIREN is comprised of a 1994 GM Saturn with the running gear removed, embedded electronic sensors, unobtrusive cameras for recording driver performance, a sound system and surrounding screens $\left(150^{\circ}\right.$ forward and $50^{\circ}$ rear fields of view), LCD projectors with image generators, and computers for scenario design, control, and data collection. All street signs and road scenarios conform to the requirements of AASHTO (American Association of State Highway and Transportation Officials) and MUTCD (Manual for Uniform Traffic Control Devices). Prior to beginning the experiment, drivers were given a 5 to 10 minute "warm-up and training" session to become familiar with the vehicle controls and to ensure a level of proficiency sufficient to proceed with the experiment.

\section{Car Following Task}

Drivers performed a car following task in which they were requested to maintain a two car length distance from the LV (as in Dastrup et al, in press). During the task, the LV velocity varied according to a sum of sine function. Table 1 provides additional information regarding the task using the standard format suggested by the Simulator Users Group (http://www.drivingwiki.org/, accessed 04/03/09). 


\section{Table 1. Car Following Scenario}

\begin{tabular}{l|l} 
Scenario & Car Following \\
\hline Description & At the start of
\end{tabular}

the start of the scenario a LV was $18 \mathrm{~m}$ in front of the participant vehicle. The LVs velocity was programmed to vary velocity following a pattern created by the sum of three sine waves (Andersen and $\mathrm{Ni}, 2005)$. After 500 meters in which the LV maintained a headway of 18 meters, the LV began to modulate its velocity according to a sum of sines function. Three sinusoids were used to create the LVs seemingly unpredictable behavior. The amplitudes of the three sinusoids were 6.072 (9.722), 2.417 (3.889), and $1.726(2.778) \mathrm{mph}(\mathrm{kph})$. The corresponding frequencies were $0.033,0.083$, and 0.117 $\mathrm{Hz}$. The phase for each sinusoid differed. The phase for the high and middle frequency sinusoids were randomly assigned a value between 0 and 1 . The low frequency sinusoid was then assigned a value that caused the sum of the three sinusoids to be zero on the first frame, thereby ensuring that the LV always started the task at a velocity of $55 \mathrm{mph}$. The random phase values caused the LVs velocity pattern to be different for each participant.

Participant Instructions Drivers were instructed to maintain a two car length headway distance while following the LV.

\begin{tabular}{l|l} 
Measures of interest & Cognitive constructs stressed: Attention, perception, vigilance, continuous visuomotor performance
\end{tabular} and risk acceptance/ risk taking.

Dependent driving variables: Following distance (mean, SD); coherence, gain, and delay are calculated using Fourier analysis (Brookhuis et al., 1994; Janacek, 2008)

- Coherence measures how well the subject vehicle matches LV velocity changes. The measure is similar to correlation with values ranging between zero to one. Higher values of coherence indicate closer relationships between the two vehicle velocities; when coherence is low (we use coherence $\leq 0.3$ ) gain and delay are not reliable. In Figure 1, the top two graphs depict examples of subjects with high coherence $(0.95$ and 0.93$)$ while the bottom two graphs depict examples of subjects with low coherence $(0.57$ and 0.54$)$.

- Gain is an amplification factor measuring the amount by which the subject overshoots or undershoots the LV velocity changes. Gain is calculated as the ratio of the spectral density of the subject vehicle velocity / spectral density of the LV velocity. Gain values greater than one indicate overreactions while values less than one are indicative of underreactions to the LV velocity changes (Andersen and Sauer, 2007). In figure 1, the bottom left graph is a rare example of an underreaction to the LV changes in velocity (0.45). The top left graph is an example of slight overshoot (1.28) and the two graphs on the right demonstrate very high values of gain (4.03 and 7.34).

- Delay is measured in seconds and indicates the time it takes for a driver to react to LV velocity changes. Delay is calculated as the phase / (frequency * \# of frames recorded per second). De Waard and Brookhuis (2000) indicate that this measure is most indicative of driving safety; a subject with slow reactions is an unsafe driver.
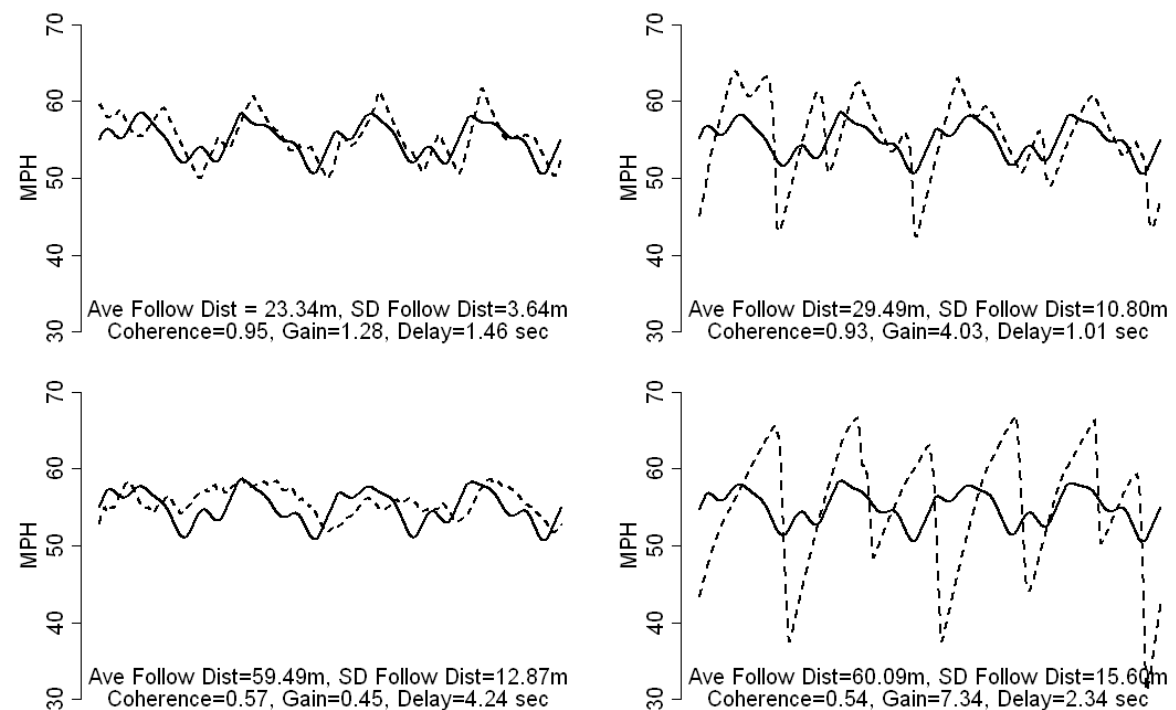

Figure 1: Demonstration of coherence, gain and delay

Data Reduction /

Variable Calculation
Following distance and velocity were recorded at $60 \mathrm{~Hz}$. A Fourier analysis derived coherence, gain and delay using the velocities of the LV and the subject vehicle. The values for gain, coherence, and delay were obtained for the frequency with the highest spectral density for the LV. 


\begin{tabular}{l|l}
\hline $\begin{array}{l}\text { Implementation } \\
\text { Variations }\end{array}$ & $\begin{array}{l}\text { Variations of the task could be done by modifying the driver instructions, changing speed parameters, } \\
\text { changing the specified following distance, etc. }\end{array}$ \\
\hline $\begin{array}{l}\text { Measurement } \\
\text { Challenges }\end{array}$ & $\begin{array}{l}\text { Some drivers may not perform the task as instructed. When performed over a longer period of time, } \\
\text { measures derived with the Fourier analysis become more stable. }\end{array}$ \\
\hline Validity & $\begin{array}{l}\text { Drivers may have different car following behavior in the real world, e.g., because of added risk and } \\
\text { different visual and vestibular cues. Instrumented vehicles could be used to study car following } \\
\text { behavior on the road, however environmental variables are less easily controlled and the safety risk is } \\
\text { greater on the road. }\end{array}$ \\
\hline Useful References & $\begin{array}{l}\text { Brookuis K, de Waard D, B. Mulder (1994) Measuring Driving Performance By Car-Following in } \\
\text { Traffic. Ergonomics, 1994 vol. 37 no. 3 427-434 }\end{array}$ \\
& $\begin{array}{l}\text { Andersen G J, Ni R (2005) The Spatial Extent of Attention During Driving. Proceedings of the } \\
\text { Third International Driving Symposium on Human Factors in Driver Assessment, Training and }\end{array}$ \\
& $\begin{array}{l}\text { Vehicle Design } \\
\text { de Waard D, Brookhuis KA. Drug Effects on Driving Performance. Annals of Internal Medicine } \\
\text { 2000 133: 656. } \\
\text { Andersen G J, Sauer C W (2007) Optical Information for Car Following: The Driving by Visual } \\
\text { Angle (DVA) Model. Human Factors, 2007, vol. 49 no. 5 pp 878-896 }\end{array}$ \\
\hline
\end{tabular}

\section{Data Reduction/Variable Calculation}

A finite Fourier transform was performed using the subject and LV velocity during the car following task to obtain coherence, gain, and delay as proposed by Brookhuis et al. (1994). The data were smoothed using a Tukey-Hanning procedure to ensure consistent estimations (Janacek, 2008). The Fourier analysis output for $n / 2$ frequencies $(n=\#$ of frames in the driving segment) between 0 and $30 \mathrm{~Hz}$ (with $30 \mathrm{~Hz}$ corresponding to the $\mathrm{n} / 2^{\text {nd }}$ frequency) included: 1 ) spectral density of the LV velocity, 2) spectral density of the subject vehicle velocity, 3) coherence, and 4) phase. Spectral density is used to identify the frequency at which periodic changes occur (Janacek, 2008). Coherence, gain (spectral density of the subject vehicle velocity / spectral density of the LV velocity), and delay (phase / (frequency * \# of frames recorded per second) at the frequencies where the periodic changes are occurring characterize how well a subject responds to the LV velocity changes (see table 1 for a more detailed description). For this study the values for gain, coherence, and delay were obtained from the frequency with the highest spectral density for the LV (the frequency output closest to $0.033 \mathrm{~Hz}$ ). There were not enough data from the smaller sine waves to conduct this analysis. This approach resembled that of Brookhuis et al. (1994) who used measures of coherence, gain, and delay obtained from the single most appropriate frequency, and differs from the methods of Andersen and Ni (2005) who averaged measures across three peaks.

In this study, a coherence of 0.3 was used to determine which subjects should be included in the analysis. Values below this threshold indicate that the participant is not engaged in the car following task. Based on this cutoff value 2 younger and 11 older participants were excluded from the analyses for gain, delay, and coherence. Older subjects showed a trend towards being less likely to reach the threshold value $(\mathrm{p}=0.067)$.

\section{RESULTS}

Coherence, delay, gain, average following distance and standard deviation of following distance (explained within Table 1) were compared between older and younger drivers using a Wilcoxon Rank Sum tests. Spearman correlations were obtained for standard deviation of following distance. The number of subjects deleted due to low coherence was compared using Fisher's exact test. 
A greater percentage of younger subjects met the coherence cutoff. Even excluding participants with coherence less than 0.3 , younger drivers showed higher median coherence than the older drivers $(0.84 \mathrm{v} 0.76, \mathrm{p}=0.0185)$, indicating that they better matched $\mathrm{LV}$ velocity changes. Younger drivers had lower gain values compared to older drivers $(1.74 \mathrm{v} 2.24, \mathrm{p}=0.031)$, suggesting that older drivers overshot in reaction to LV velocity changes. These older drivers also showed a trend toward quicker response (shorter median delay) to the changes in LV velocity ( $2.22 \mathrm{~s} \mathrm{v} 3.06 \mathrm{~s}, \mathrm{p}=0.077)$, suggesting that older drivers are more focused on the carfollowing task.

Mean and SD following distance were calculated for all participants (including those with coherence less than 0.3). Younger drivers maintained a shorter median headway (following) distance than older drivers $(32.91 \mathrm{~m} \vee 59.95 \mathrm{~m}$; $\mathrm{p}<0.0001)$ and showed less variability in following distance $(6.44 \mathrm{~m} v 15.66 \mathrm{~m} ; \mathrm{p}<0.0001)$. Table 2 lists the results for the car following measures.

Table 2. Results for car following measures (mean \pm SD (median) and Wilcoxon Ranked sign test p values)

\begin{tabular}{l|l|c|l} 
& \multicolumn{1}{|c}{ Younger } & Older & p-value \\
\hline Coherence & $0.80 \pm 0.17(0.84)$ & $0.69 \pm 0.20(0.76)$ & $\mathbf{0 . 0 1 9}$ \\
\hline Delay (seconds) & $3.58 \pm 2.39(3.06)$ & $3.13 \pm 2.94(2.22)$ & $\mathbf{0 . 0 7 7}$ \\
\hline Gain & $2.45 \pm 3.24(1.74)$ & $2.66 \pm 1.52(2.24)$ & $\mathbf{0 . 0 3 1}$ \\
\hline Mean following distance (m) & $44.42 \pm 43.23(32.91)$ & $82.45 \pm 64.26(59.95)$ & $<\mathbf{0 . 0 0 0 1}$ \\
\hline $\begin{array}{l}\text { Standard deviation of following } \\
\text { distance (m) }\end{array}$ & $12.80 \pm 24.57(6.44)$ & $20.15 \pm 16.21(15.66)$ & $<\mathbf{0 . 0 0 0 1}$
\end{tabular}

Table 3 shows the correlations of average following distance with SD car following distance, coherence, delay, and gain. Coherence has a high negative correlation with average following distance in both driver groups, suggesting that drivers match LV velocity changes more accurately when following more closely. Delay correlated strongly with average following distance. Compared to younger drivers, older drivers followed the LV at further distances and showed a trend toward faster responses to LV velocity changes. However, like younger drivers, older drivers who followed closer responded faster.

Table 3. Spearman correlations with Average Following Distance

\begin{tabular}{l|l|l} 
& Older & Younger \\
\hline $\begin{array}{l}\text { SD car following } \\
\text { distance (m) }\end{array}$ & $0.756 * * *$ & $0.848 * * *$ \\
\hline Coherence & $-0.859 * * *$ & $-0.718 * * *$ \\
\hline Delay (seconds) & $0.819 * * *$ & $0.706 * * *$ \\
\hline Gain & -0.096 & 0.244 \\
$+\mathrm{p}<.1 * \mathrm{p}<.05 * * \mathrm{p}<.01 * * * \mathrm{p}<.001$ &
\end{tabular}

While average car following distance is important, it does not fully characterize driver performance. In figure 1, the top two subjects take greater potential risk by adopting shorter following distances compared to the bottom two subjects, yet they have "better" values of coherence (higher), gain (closer to 1), and delay (lower), indicating greater ability to accommodate the potential risk. Another possibility is that the LV is more salient when the driver follows closer behind because it subtends a greater visual angle (Donmez, Boyle, \& Lee, 2008). The bottom two subjects adopt similar following distances, yet differ in their accommodations based on values of delay and gain. 


\section{DISCUSSION}

The results of the study showed differences in car following behaviors between younger and older drivers. Older drivers matched LV velocity changes less closely and followed further behind the LV compared to younger drivers. Greater car following distances may represent a compensatory behavior that serves to reduce older driver risk. In contrast, younger drivers follow closer and respond later than older drivers. The propensity to follow closer has the potential to increase crash involvement in real-world driving (Evans \& Wasielewski, 1982).

Differences in car following between younger and older drivers depend on a variety of cognitive and behavioral factors. These include differences in driver vision, vigilance, cognition, judgment, motor abilities, and acceptance of risk. In this regard, standard measures of car following such as average and SD of following distance are useful, yet they may fail to capture key aspects of driver control and behavior.

Drivers with equivalent SD of following distance may vary greatly in driving style and risk taking. In this study of younger and older drivers, measures derived from Fourier analysis gave additional information about the nature and quality of car following behavior beyond SD of following distance. The results are broadly compatible with findings from a recent study of car following in younger drivers who had used MDMA and THC and non drug users (Dastrup et al, in press), where the delay measure was the primary outcome of interest.

The willingness to follow a vehicle at shorter headways, aggressive instances of which are known as "tailgating", may increase crash involvement in real-world driving (Evans \& Wasielewski, 1983). In the current driving simulator study, younger driver may have been willing to follow the LV at shorter distances, in part because they feel more comfortable with the risks and task demands in real and virtual environments than older drivers do. Alternatively, they may have less experience dealing with erratic speed variations by a LV, in line with reports of less hazard awareness of younger drivers tested in other driving situations (Pradhan, et al., 2005). Future studies of car following behavior can address these possibilities by further manipulating task difficulty instructions to follow a LV more or less closely.

\section{ACKNOWLEDGEMENTS}

Special thanks to Dick de Waard, John Andersen, Ben Mulder, Rui Ni and Joseph Cavanaugh for correspondence on the analysis of car following performance data.

Supported by NIA AG17177 and NIDA DA017341.

\section{REFERENCES}

Andersen, G.J., Ni, R. (2005). The spatial extent of attention during driving. Proceedings of the Third International Driving Symposium on Human Factors in Driver Assessment, Training and Vehicle Design, Rockport Maine, 403-408.

Andersen G J, Sauer C W (2007) Optical information for car following: The driving by visual angle (DVA) model. Human Factors, 49(5), 878-896. 
Brackstone, M, McDonald M (1999) Car-following: a historical review. Transportation Research Part F, 2, 181-196.

Brookuis, K., de Waard, D. and Mulder, B. (1994). Measuring driving performance by carfollowing in traffic, Ergonomics, 37(3), 427-434.

Dastrup, E., Lees, M., Bechara, A., Dawson, J.D., Rizzo, M. (in press). Risky car following in abstinent users of MDMA.

de Waard, D., Brookhuis, K.A. (2000). Drug effects on driving performance. Annals of Internal Medicine, 133, 656.

Dingus, T.A., Jahns, S.K., Horwitz, A.D., Knipling, R.R (1998). Human factors design issues for crash avoidance systems. In T.A. Dingus (Ed.), pp. 55-94. Mahwah, NJ: Erlbaum.

Dingus, T.A., McGehee, D. V., Manakkal, N., Jahns, S.K., Carney, C., Hankey, J.M. (1997). Human factors field evaluation of automotive headway maintenance/collision warning devices. Human Factors, 39, 216-229.

Donmez, B., Boyle, L., \& Lee, J. D. (2008). Accounting for the covariate effects in driving simulator studies. Theoretical Issues in Ergonomics Science, 9(3), 189-199.

Evans, L, and Wasielewski, P. (1982). Do accident-involved drivers exhibit riskier everyday driving behavior. Accident Analysis and Prevention, 14, 57-64.

Evans, L. Wasielewski, P. (1983). Risky driving related to driver and vehicle characteristics. Accident Analysis and Prevention, 15, 121-136.

Pradhan, A. K., Hammel, K. R., DeRamus, R., Pollatsek, A., Noyce, D. A., \& Fisher, D. L. (2005). Using eye movements to evaluate effects of driver age on risk perception in a driving simulator. Human Factors, 47(4), 840-852.

Janacek, G. J. (2008). Spectral Analysis. Encyclopedia of Biostatistics 2nd Edition, pp. 1-13. John Wiley \& Sons, Inc. New York.

Knipling, R.R., Mironer, M., Hendricks, D.L., Tijerina, L., Everson, J., Allen, J.C., Wilson, C. (1993). Assessment of IVHS countermeasures for collision avoidance: Rear-End crashes Washington, DC: National Highway Traffic Safety Administration.

National Highway Traffic Safety Administration (2007). 2005 Traffic safety facts annual report. Washington, DC: Department of Transportation, 2007. DOT HS 810631.

Simulator Users Group. http://www.engineering.uiowa.edu/simusers/Scenarios/scenarios.htm. Accessed 7/27/2008.

Rizzo, M., Shi, Q., Dawson, J., Anderson, S.W., Kellison, I., and Pietras, T.A. (2005). Stops for cops: Impaired response implementation in older drivers with cognitive decline.

Transportation Research Record: Journal of the Transportation Research Board, 1922, 1-8.

Wang. J., Knipling, R.R., Goodman, M.J. (1996). The role of driver inattention in crashes; new statistics from the 1995 crashworthiness data system (CDS). 40th Annual Proceedings of the Association for the Advancement of Automotive Medicine, Association for the Advancement of Automotive Medicine, Des Plaines, Iowa, 377-392. 